\title{
Epigenetic bivalent marking is permissive to the synergy of HDAC and PARP inhibitors on TXNIP expression in breast cancer cells
}

\author{
FEDERICA BALDAN $^{1}$, CATIA MIO ${ }^{1}$, ELISA LAVARONE ${ }^{1}$, CARLA DI LORETO $^{1,2}$, \\ FABIO PUGLISI $^{3}$, GIUSEPPE DAMANTE ${ }^{1,4}$ and CINZIA PUPPIN ${ }^{1}$ \\ ${ }^{1}$ Department of Medical and Biological Sciences, University of Udine; ${ }^{2}$ Institute of Anatomical Pathology, \\ ${ }^{3}$ Department of Oncology and ${ }^{4}$ Institute of Medical Genetics, University Hospital 'S. Maria della Misericordia' Udine, Italy
}

Received September 26, 2014; Accepted December 3, 2014

DOI: $10.3892 /$ or.2015.3873

\begin{abstract}
Studies on stem cell differentiation led to the identification of paused genes, characterized by the contemporary presence of both activator and repressor epigenetic markers (bivalent marking). TXNIP is an oncosuppressor gene the expression of which was reduced in breast cancer. In the present study, we evaluated whether the concept of epigenetic bivalent marking can be applied to TXNIP gene in breast cancer cells. Using chromatin immunoprecipitation (ChIP), three histone modifications were investigated: two associated with transcriptional activation, lysines 9-14 acetylation of $\mathrm{H} 3$ histone (H3K9K14ac) and lysine 4 trimethylation of $\mathrm{H} 3$ histone (H3K4me3), and one associated with transcriptional silencing, lysine 27 trimethylation of $\mathrm{H} 3$ histone (H3K27me3). According to the bivalent marking model, TXNIP gene appears to be paused in MDA157 cells (markers of active and repressed transcription are present), but are definitively silenced in MDA468 cells (presence of only markers of transcription repression). This was proven by evaluating TXNIP mRNA and protein levels after the treatment of cell lines with a histone deacetylase inhibitor (SAHA) and a poly-ADPribose polymerases inhibitor (PJ34). In MDA157 cells, SAHA and PJ34 showed a synergistic effect: a large increment was observed in TXNIP mRNA and protein levels. By contrast, in MDA468 cells, synergy between the two compounds was not observed. Therefore, the pausing epigenetic signature was permissive for synergy between SAHA and PJ34 on TXNIP gene expression. The synergy between SAHA and PJ34 on TXNIP expression was associated with variation in cell viability and apoptosis. In MDA157 cells, but not in MDA468 cells, combined treatment of SAHA and PJ34 induced a decrease in cell viability and an increase of apoptosis. Thus, our data support the hypothesis that TXNIP is an effective target for the treatment of breast cancer.
\end{abstract}

Correspondence to: Professor Giuseppe Damante, Dipartimento di Scienze Mediche e Biologiche, Piazzale Kolbe 4, I-33100 Udine, Italy

E-mail: giuseppe.damante@uniud.it

Key words: HDAC, PARP1, epigenetics, cell proliferation

\section{Introduction}

TXNIP (also known as vitamin D3-upregulated protein or thioredoxin-interacting protein), was originally isolated in HL60 leukemia cells treated with 1,25-dihydroxyvitamin D3 (1). TXNIP has subsequently been identified as a key modulator of the redox system. It binds to the active cysteine residue of thioredoxin (TRX) and inhibits its antioxidative function (2). In addition, TXNIP can act independently of its binding to TRX, contributing to inhibition of cell growth by arresting the domain-mediated suppression of glucose uptake and metabolic reprogramming $(3,4)$.

TXNIP is important in cell growth and cancer, acting as a tumor suppressor gene. Downregulation or loss of TXNIP expression has been shown in various tumors including renal, breast, lung, gastric, colon and hepatocellular carcinoma (5). Genetic alterations of TXNIP are not common, and its aberrant expression in cancer has been mainly regulated via post-transcriptional and translational mechanisms. It seems that cancer cells develop various ways, such as hypermethylation, histone deacetylation, histone methylation and post-translational inhibition by miRNA, to inactivate TXNIP expression, indicating the importance of its repression in tumorigenesis (6).

Altered TXNIP expression may be associated with disease progression and poor prognosis (7-9). The role of TXNIP in breast cancer has been previously investigated. A lower expression of TXNIP mRNA was identified in breast cancer tumors as compared to matched normal controls (10). Cadenas et al demonstrated that a low TXNIP expression was associated with worse prognosis in breast cancer, while a higher expression of TXNIP was associated with longer metastasis-free interval (7). In terms of breast cancer cell biology, Butler et al demonstrated that ectopic expression of TXNIP in MCF7 cells induced cell senescence (10).

Studies on stem cells led to the identification of paused genes (11). These genes are characterized by the contemporary presence of activator and repressor epigenetic markers (bivalent marking). These markers are based on histone post-translational modifications; for example, lysine 4 trimethylation of $\mathrm{H} 3$ histone (H3K4me3) was associated with transcriptional activation, while lysine 27 trimethylation of $\mathrm{H} 3$ histone (H3K27me3) was associated with transcriptional silencing $(12,13)$. Thus, a bivalent marking is typical of paused 
Table I. Primers used for real-time PCR analysis.

\begin{tabular}{llll}
\hline Gene name & \multicolumn{1}{c}{ Forward } & \multicolumn{1}{c}{ Reverse } & Probe \\
\hline TXNIP & GTCAGTCACTCTCAGCCATAGCA & CACACTTTCTGGCTGTAATAACTCTCA & \\
$\beta$-actin & TTGTTACAGGAAGTCCCTTGCC & ATGCTATCACCTCCCCTGTGTG & \\
TXNIP & & & \\
promoter & GAGCGCAACAACCATTTTCC & GAGCCCGACCAATCAGTGA & FAM-TGTCCACGCGCCACAGCGAT \\
\hline
\end{tabular}

genes, i.e., genes that are expressed at low levels but that can be expressed at high levels following differentiation or by external stimulation. By contrast, genes in which only markers of transcriptional silencing are detected are definitively silenced.

The epigenetic concept of paused genes has been generated by studying epigenetic marking and gene expression during differentiation of stem cells (11). However, if the bivalent marking hypothesis of paused genes is true for the gene expression of cancer cells, it may be useful for delineation of new therapeutic strategies. For example, the expression of paused oncosuppressor genes may be reactivated by external stimulation more easily than the expression of oncosuppressor genes that are definitively silenced.

Treatment of breast cancer is challenging as its heterogeneity may be due to the differential expression of estrogen receptor (ER), progesterone receptor (PR) and amplification of HER $2 /$ neu. Therapeutic options are significantly affected by the expression of the three markers (14). Triple-negative breast cancer (TNBC), characterized by the absence of $E R$, $P R$ and HER-2 gene expression, is an important clinical challenge because it does not respond to endocrine or monoclonal antibody anti HER-2 therapies.

In the present study, the hypothesis that epigenetic bivalent marking defines the possibility for re-activation of TXNIP gene expression was evaluated in TNBC-derived cell lines.

\section{Materials and methods}

Cell lines and immunohistochemistry. MDA157 and MDA468 human cell lines derived from triple negative breast cancer, were grown in DMEM medium supplemented with $10 \%$ fetal bovine serum (Gibco Invitrogen, Milan, Italy), $2 \mathrm{mM}$ L-glutamine (EuroClone, Milan, Italy) and $50 \mathrm{mg} / \mathrm{ml}$ gentamicin (Gibco Invitrogen) in a humidified incubator $\left(5 \% \mathrm{CO}_{2}\right.$ in air at $\left.37^{\circ} \mathrm{C}\right)$.

Core biopsies of triple negative breast tumors were examined. The tissues were formalin-fixed for 16-24 h. Formalin-fixed paraffin tissue sections $(5 \mu \mathrm{m})$ mounted on Superfrost slides (Surgipath, Richmond, IL, USA) were immunohistochemically stained, by using the peroxidase/DAB Plus Dako Real EnVision ${ }^{\mathrm{TM}}$ detection system (Dako A/S, Glostrup, Denmark). Antigen retrieval was performed in a water bath at $98^{\circ} \mathrm{C}$ with $0.01 \mathrm{M}$ citrate buffer, at $\mathrm{pH} 6.0$ for $40 \mathrm{~min}$. Endogenous peroxidase activity was blocked by incubation in the Peroxidase Block solution (Dako A/S) for 10 min. Primary rabbit polyclonal antiserum to TXNIP (Abcam, Cambridge, UK) diluted at 1:100 was applied and incubated for $60 \mathrm{~min}$ at room temperature. After being washed, the slides were incubated with the peroxidase/DAB Plus Dako Real EnVision ${ }^{\mathrm{TM}}$ detection system (Dako) according to the manufacturer's instructions. For reaction visualization, 3,3'-diaminobenzidine tetrahydrochloride was used as chromogen. The sections were counterstained with Mayer hematoxylin. Normal breast tissue sections were used as a positive control, whereas the negative control was performed by replacing the primary antibody with PBS. Nuclear and cytoplasmic immunostaining were evaluated by using light microscopy, in which the entire section was scanned at high-power magnification (x400). In the case of cell cultures, MDA157 cells, treated or not with SAHA $3 \mu \mathrm{M}$ and PJ34 $5 \mu \mathrm{M}$ alone or in combination, were immediately fixed with ethanol containing 5\% glacial acetic acid for $10 \mathrm{~min}$ at $4^{\circ} \mathrm{C}$, and rinsed with $0.1 \%$ saponin (Sigma Chemical Co., St. Louis, MO, USA) in phosphate-buffered saline (PBS). This PBS-saponin solution was also used for all subsequent washing steps. The cultures were incubated overnight at $4^{\circ} \mathrm{C}$ with rabbit antiserum to TXNIP, diluted at 1:100. After washing the Dako Real EnVision ${ }^{\mathrm{TM}}$ detection system kit, peroxidase/DAB ${ }^{+}$, rabbit/mouse K5007 (Dako) was used. Peroxidase activity was detected with 3,3'-diaminobenzidine tetrahydrochloride followed by haematoxylin counterstaining.

Chromatin immunoprecipitation (ChIP). In order to perform ChIP assay, MDA157 and MDA468 cells treated or not for $72 \mathrm{~h}$ with SAHA $3 \mu \mathrm{M}$ and PJ34 $5 \mu \mathrm{M}$, were subjected to cross-linking by using $1 \%$ formaldehyde $\left(10 \mathrm{~min}\right.$ at $\left.37^{\circ} \mathrm{C}\right)$. The cells were then scraped in PBS with proteinase inhibitors and resuspended in cell lysis buffer (1\% SDS, $10 \mathrm{mM}$ EDTA, $50 \mathrm{mM}$ Tris- $\mathrm{HCl} \mathrm{pH} 8.1$ and protease inhibitors). The samples were subjected to sonication and diluted 10 -fold with dilution buffer (0.01\% SDS, 1.1\% Triton X-100, 1.2 mM EDTA, $16.7 \mathrm{mM}$ Tris- $\mathrm{HCl} \mathrm{pH} 8.1,167 \mathrm{mM} \mathrm{NaCl}$ ); $8 \%$ of this was saved as total input.

The samples were incubated for preclearing with Dynabeads Protein A (Gibco Invitrogen). For immunoprecipitation, th esamples were incubated overnight with $10 \mu \mathrm{g}$ of rabbit polyclonal anti-acetyl-histone $\mathrm{H} 3$ antibody (Upstate Temecula, CA, USA) or anti-histone H3 trimethyl Lys4 antibody or anti-histone H3 trimethyl Lys27 antibody (both from Active Motif) or rabbit polyclonal anti-histone H3 trimethyl K9 antibody (Abcam). The following day, samples and negative controls (samples with purified Rabbit IgG, Millipore) were added with Dynabeads Protein A.

After being washed, the immunocomplexes were eluted from beads with elution buffer (1\% SDS, $0.1 \mathrm{M}, 50 \mathrm{mM}$ $\mathrm{NaHCO}_{3}$ ), crosslinks were reverted by heating and the samples were treated with proteinase K. DNA was purified with phenol/ chloroform extraction followed by ethanol precipitation, which was used as a template in quantitative absolute PCR, whose reaction primers are presented in Table I. After quantitative 
A

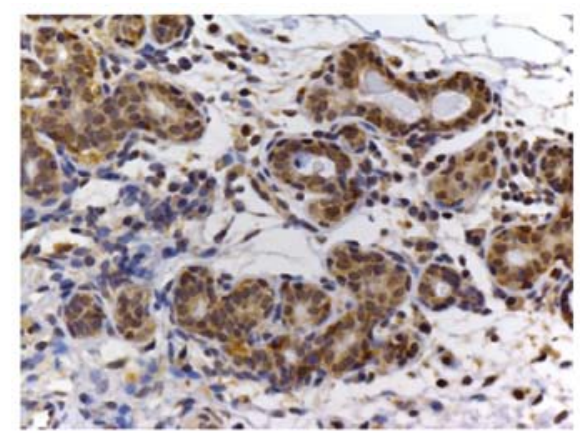

C

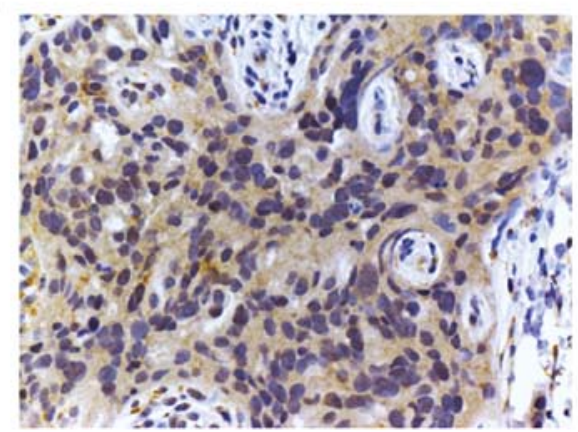

B

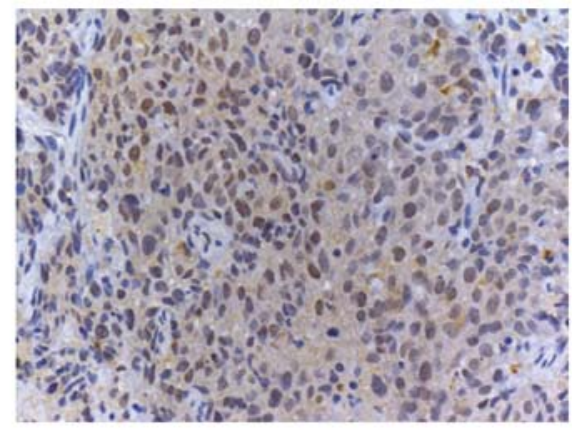

D

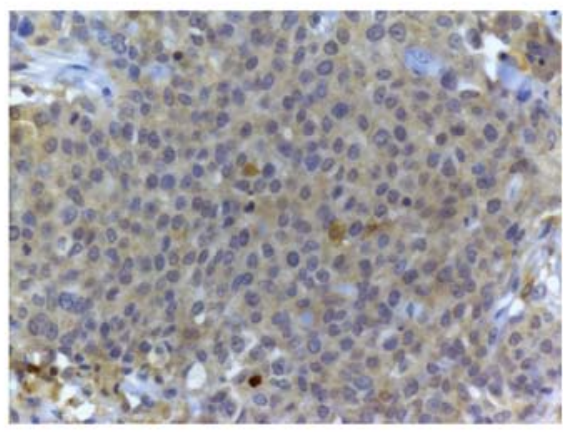

Figure 1. Immunohistochemical detection of TXNIP in normal breast tissue and triple-negative breast cancer. Immunohistochemistry was performed as described in Materials and methods. (A) TXNIP expression in normal breast tissue. (B-D) TXNIP expression in triple negative breast cancer.

PCR reactions, the acetylated or methylated $\mathrm{H} 3$ levels were determined as a ratio of signals recorded after and prior to (input) immunoprecipitation.

Quantitative RT-PCR. Total RNA from cell lines, treated for $72 \mathrm{~h}$ with SAHA $3 \mu \mathrm{M}, \mathrm{PJ} 345 \mu \mathrm{M}$ alone or in combination, were extracted with an RNeasy mini kit, according to the manufacturer's instructions (Qiagen, Hilden, Germany). Total RNA (500 ng) was reverse transcribed to cDNA using random exaprimers and MMLV reverse transcriptase (Invitrogen). Quantitative PCRs were performed using PlatinumSYBR-Green qPCR supermix (Life Technologies) or TaqMan Universal PCR master mix (Applied Biosystems, Foster City, CA, USA) with the ABI Prism 7300 sequence detection systems (Applied Biosystems). The $\Delta \Delta \mathrm{Ct}$ method, by means of the SDS software (Applied Biosystems), was used to calculate the mRNA levels. Oligonucleotide primers for TXNIP and for the endogenous control, $\beta$-actin, were purchased from Sigma (Table I).

Protein extraction and western blotting. In order to detect the TXNIP protein, total protein extraction was performed. To this purpose, MDA157 and MDA468 cells, cultured for $72 \mathrm{~h}$ in growth medium, in presence or absence of SAHA $3 \mu \mathrm{M}$, PJ34 $5 \mu \mathrm{M}$ alone or in combination, were harvested by scraping and lysed with Laemmli/SDS lysis buffer (1.0 M Tris, pH 6.8, 2\% SDS, $0.001 \%$ bromophenol blue, $10 \%$ glycerol, $2 \% \beta-\mathrm{ME})$. The samples were then subjected to sonication.

For the western blot analysis, the proteins were electrophoresed on $10 \%$ SDS-PAGE and then transferred to nitrocellulose membranes, which were saturated with $5 \%$ non-fat dried milk in PBS $/ 0.1 \%$ Tween-20. The membranes were then incubated overnight with rabbit polyclonal anti-TXNIP antibody or anti$\beta$-actin antibody (both from Abcam). The following day, the membranes were incubated for $2 \mathrm{~h}$ with anti-rabbit immuno- globulin coupled with peroxidase (Sigma-Aldrich). The blots were developed using Chemidoc XRS (Bio-Rad, Hercules, CA, USA) with the chemiluminescence procedure (Amersham Biosciences, Buckinghamshire, UK).

Cell viability and apoptosis assays. To test cell viability the MTT assay was used as previously described (15). The cells were seeded in 96-well plates in $200 \mu \mathrm{l}$ medium. The following day, the growth medium was replaced with fresh medium (untreated cultures) or with medium containing SAHA $3 \mu \mathrm{M}$ (Cayman Chemical, Ann Harbor, MI, USA) and PJ34 $5 \mu \mathrm{M}$ (Merck Chemicals Ltd., Nottingham, UK) alone or in combination, and the plates were incubated for $72 \mathrm{~h}$. Experimental points were run in quadruplicate.

Caspase 3/7 activity was measured as a marker of apoptosis using the ApoOne Homogeneous Caspase 3/7 assay kit (Promega, Milan, Italy) according to the manufacturer's instructions. Experimental points were run in triplicate.

Statistical analysis. TXNIP mRNA and protein levels, cell viability and apoptosis levels were expressed as the mean \pm SD values, and significances were analyzed with the t-test performed with GraphPad software for science (San Diego, CA, USA).

\section{Results}

Immunohistochemical detection of TXNIP in normal breast tissue and triple-negative breast cancer. Identification of specific therapeutic options for TNBC remain a challenge. Therefore, we focused our investigation on this type of cancer. TNBC tissues were immunostained and, despite a certain degree of heterogeneity, a decrease of TXNIP expression was detected. Fig. 1 shows some exemplifying cases: compared to 
$\mathbf{A}$

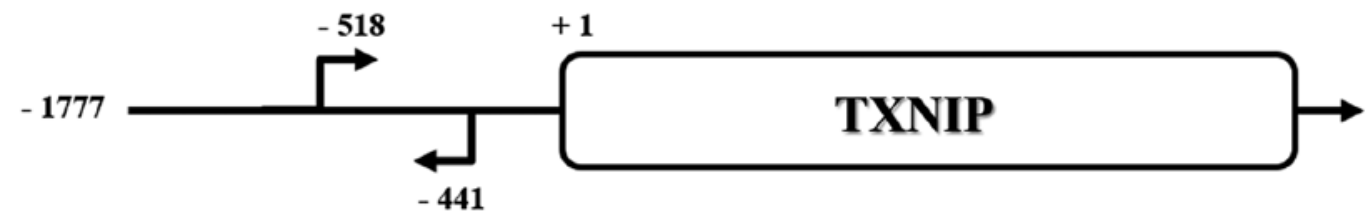

B
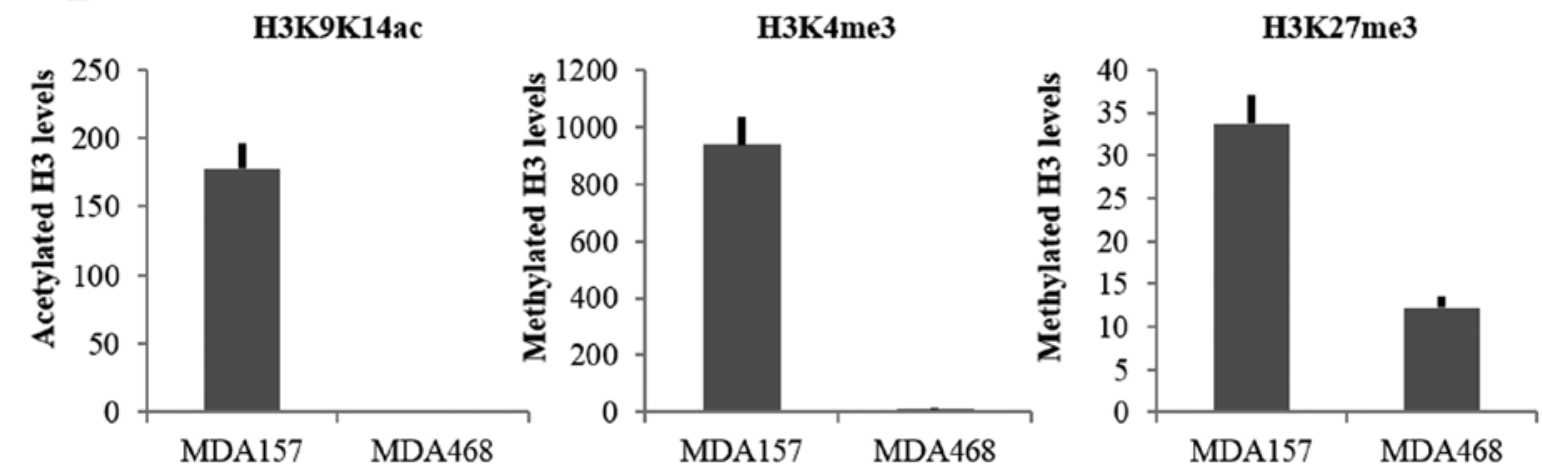

Figure 2. Histone post-translational modifications on TXNIP promoter. (A) Schematic structure of TXNIP promoter; arrows indicate positions of primers used for amplification of the region assayed by ChIP. (B) Basal H3K9K14ac, H3K4me3 and H3K27me3 levels on TXNIP promoter in MDA157 and MDA468 cells. Each bar is the mean value \pm SD of three different determinations.

normal breast tissue (A), TNBC shows a weak cytoplasmic signal (B-D). However, in the majority of cells no nuclear staining was identified (C and D).

Histone post-translational modifications on TXNIP promoter. In order to evaluate the bivalent marking hypothesis, we used the MDA157 and MDA468 TNBC-derived cell lines. Histone modifications associated with transcriptional activation or silencing were evaluated on TXNIP promoter (Fig. 2A). By using ChIP, three histone modifications were investigated: two associated to transcriptional activation, histone $\mathrm{H} 3$ lysines 9-14 acetylation (H3K9K14ac) and histone H3 lysine 4 trimethylation (H3K4me3), and one associated with transcriptional silencing, histone $\mathrm{H} 3$ lysine 27 trimethylation (H3K27me3) (12,13). As shown in Fig. 2B, a difference was identified between the two cell lines: in MDA157 all three markers were detected, whereas in MDA468 only H3K27me3 was detectable. Thus, in MDA157 cells the bivalent marking was observed in the promoter of TXNIP gene, suggesting that its expression was prone to be activated. Instead, in MDA468 cells only the marker associated with gene silencing was detectable in the TXNIP promoter, suggesting that transcription of this gene was totally blocked. The basal levels of global histone modifications (evaluated by western blotting) in MDA157 and MDA468 cells has been previously identified (16). When results shown in Fig. 2B were compared to those data, differences of H3K9K14ac, H3K4me3 and H3K27me3 observed in the TXNIP promoter were not due to differences of global levels of these modifications. In particular, global levels of H3K27me3 were much lower in MDA468 than in MDA157. Therefore, in terms of relative amount, marker levels associated with gene silencing (H3K27me3) in MDA468 were much higher than those in MDA157.
Effects of SAHA and PJ34 on TXNIP gene expression and histone post-translational modifications. To evaluate the possibility of activating TXNIP gene expression in MDA157 we used two different compounds. The first one was the histone deacetylase (HDAC) inhibitor suberoylanilide hydroxamic acid (SAHA), already FDA-approved for the treatment of several neoplastic diseases $(17,18)$, which is able to induce TXNIP expression in transformed cells, including solid tumors and leukemia, but not in normal cells $(19,20)$. The second compound was PJ34, a poly-ADP-ribose polymerases (PARP) inhibitor. PARP is involved in numerous processes that are vital for cells. In particular, PARP proteins are activated by the presence of DNA damage, leading to poly-ADP-ribosylation of proteins involved in DNA repair, genome integrity, regulation of transcription, proliferation and apoptosis (21). In Fig. 3A, the effects of either compound on TXNIP gene expression are shown. Basal levels of TXNIP mRNA levels were higher in MDA157 than in MDA468 cells. In MDA157 cells, treatment with SAHA and PJ34 induced an increase of TXNIP mRNA levels of 15- and 10-fold, respectively. When, in this cell line, the two compounds were used together, a marked increase of TXNIP mRNA levels was observed (140-fold over basal values). Minimal effects were instead observed in MDA468 cells both with single compounds and in combination. In this cell line, TXNIP mRNA values remained markedly below the basal values observed in MDA157 cells. Thus, only the paused cell line (MDA157) was responsive to treatment with SAHA and PJ34 alone and synergy was observed when the compounds were employed in combination. By contrast, MDA468 TXNIP gene expression appeared almost insensitive to treatments, with only PJ34 having a very modest effect.

Subsequent to the synergy observed in MDA157 cells, we tested whether the combined use of SAHA and PJ34 modi- 
$\mathbf{A}$

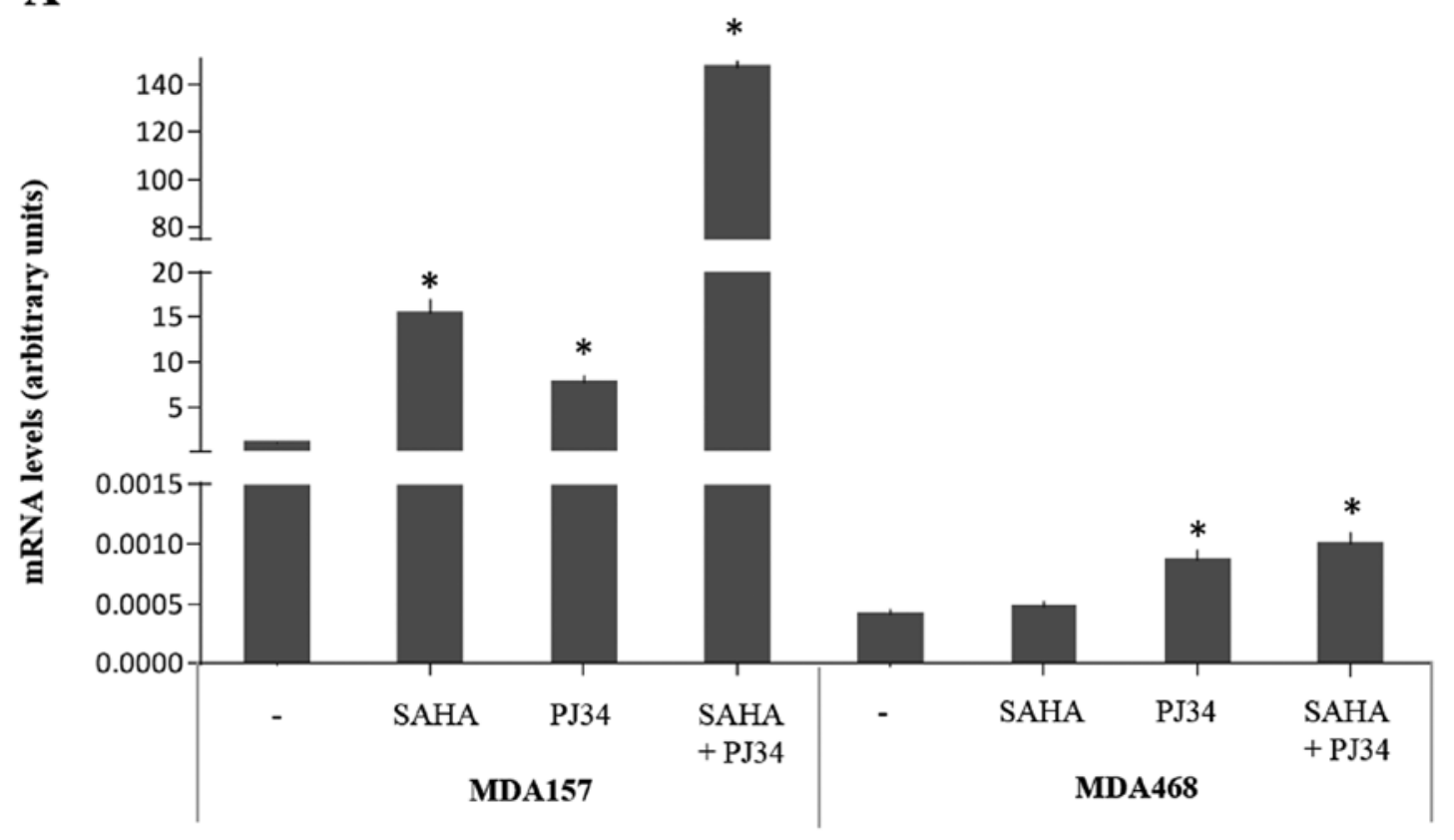

B

H3K9K14ac

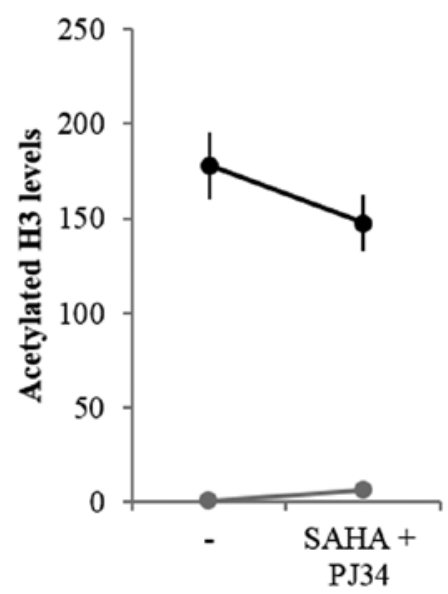

H3K4me3

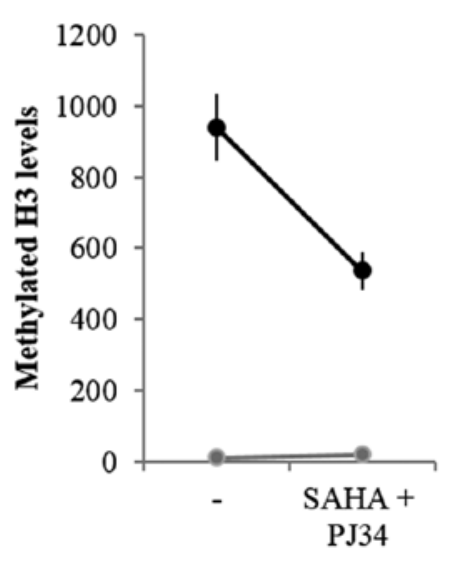

H3K27me3

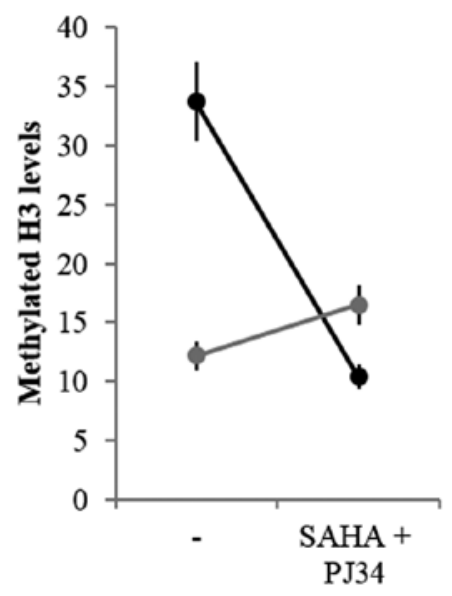

$\longrightarrow$ MDA157 - - MDA468

Figure 3. Effects of SAHA and PJ34 on TXNIP gene expression and histone post-translational modifications. (A) Effects on TXNIP mRNA levels. MDA157 and MDA468 cells were treated or not with SAHA $3 \mu \mathrm{M}$ and PJ34 $5 \mu \mathrm{M}$ alone or in combination for $72 \mathrm{~h}$. Each bar is the mean value \pm SD of three independent determinations. *Significant difference as compared to basal values $(\mathrm{P}<0.05)$. (B) Effect of SAHA and PJ34 combined treatment on H3K9K14ac, H3K4me3 and H3K27me3 levels of TXNIP promoter. The cells were treated or not for $72 \mathrm{~h}$ with SAHA $3 \mu \mathrm{M}$ and PJ34 $5 \mu \mathrm{M}$ and then subjected to ChIP assays. Black and grey circles indicate MDA157 and MDA468 cells, respectively. Each circle is the mean value \pm SD of three independent determinations.

fied histone post-translational modifications at the TXNIP promoter level. As shown in Fig. 3B, a decrease for all three post-translational modifications was observed in the MDA157 cells. However, this decrease was minimal for $\mathrm{H} 3$ acetylation but marked for H3K27me3. In MDA468, either marker associated with transcriptional activation remained almost undetectable (H3K9K14ac or H3K4me3), while the marker associated with silencing was increased. Thus, the combination of SAHA and PJ34 did not erase the silencing epigenetic signature in MDA468 cells.
Effects of SAHA and PJ34 on TXNIP protein level. To verify whether the TXNIP mRNA increase was associated with enhancement of TXNIP protein expression, a western blot analysis was performed. As shown in Fig. 4A, the MDA468 cell line did not express TXNIP protein in detectable amounts, either in basal conditions or after stimulation with SAHA and PJ34 alone or in combination. The MDA157 cell line showed an extremely low basal TXNIP protein expression, which increased following SAHA treatment and this increment was higher with the combination of SAHA and PJ34. These find- 
$\mathbf{A}$

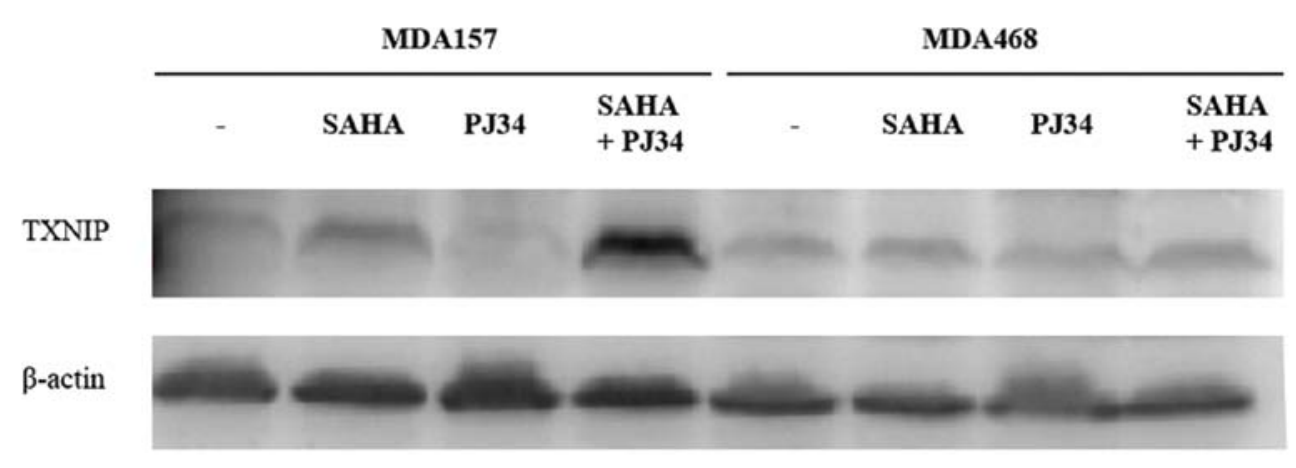

B
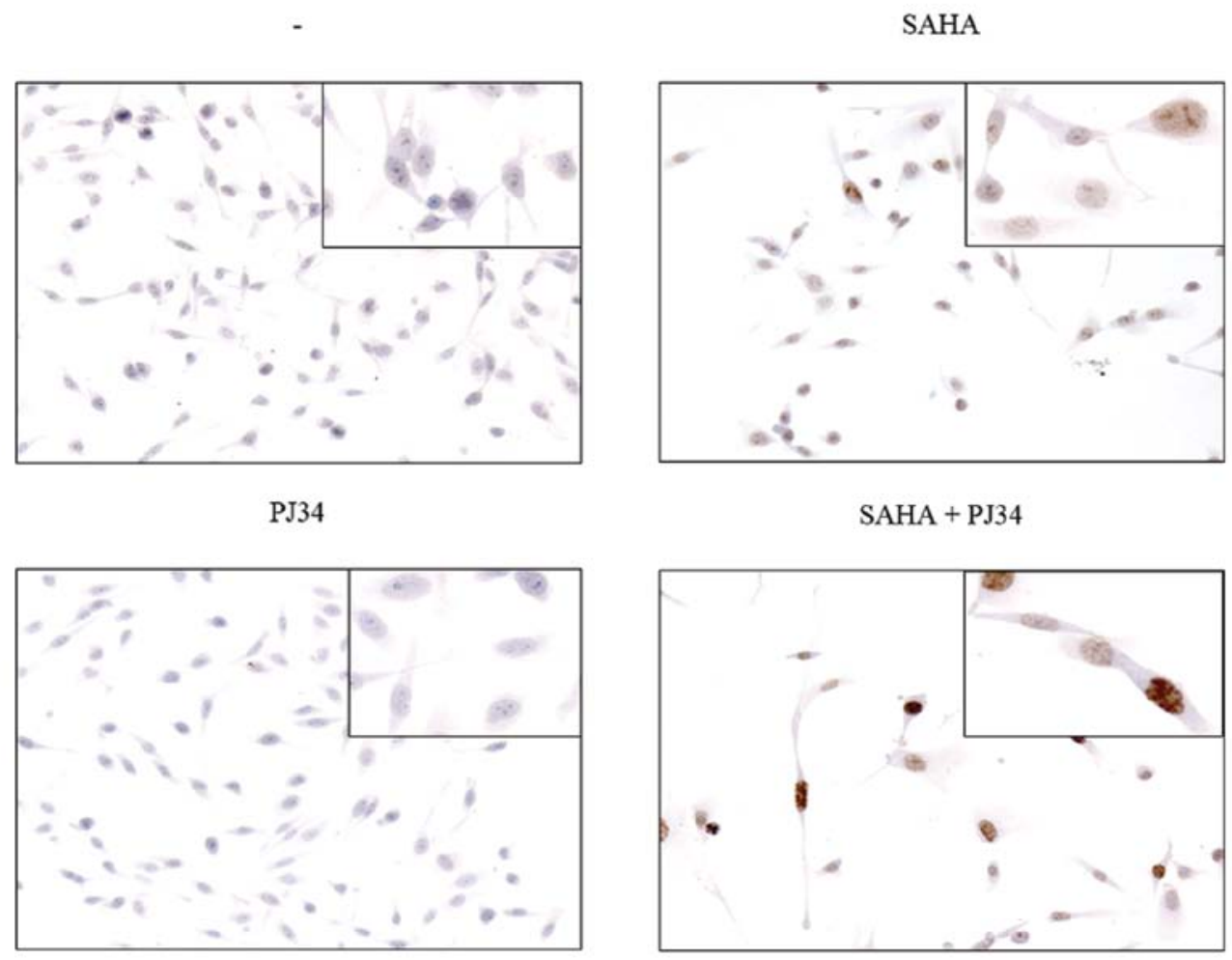

Figure 4. Effects of SAHA and PJ34 on TXNIP protein level. (A) Western blot analysis of TXNIP expression in MDA157 and MDA468 cells treated or not with SAHA $3 \mu \mathrm{M}$ and PJ34 $5 \mu \mathrm{M}$ alone or in combination for $72 \mathrm{~h}$. (B) Immunohistochemical staining of MDA157 cells. The brown signal indicates TXNIP expression.

ings were also supported by immunohistochemical analysis of MDA157 cells. In Fig. 4B, the TXNIP protein shows nuclear localization, which was weakly incremented following SAHA treatment, while no variation was observed with PJ34. The combined addition of SAHA and PJ34 markedly increased TXNIP protein levels.

Effects of HDAC and PARP inhibitors on cell viability and apoptosis in MDA157 and MDA468 cells. In various tumor tissues, the increase of TXNIP expression was correlated with growth inhibition and induction of apoptosis $(19,22)$. Therefore, we examined whether such a correlation is present in our experimental system. We evaluated whether SAHA and PJ34 cocktail may have a biological effect in terms of cell proliferation and apoptosis. In particular, cell viability was assessed in the two lines after treatment with SAHA and PJ34 alone or in combination, for $72 \mathrm{~h}$ (Fig. 5A). In the two cell lines, the treatment with SAHA alone determines a moderate decrease in viability compared to untreated cells. The effect of PJ34, however, appeared to be limited in the two lines, with a slight increase and decrease in MDA157 and MDA468 cell viability, respectively. The combination of the two compounds shows a decrease in growth compared to untreated cells, which was significantly higher in MDA157 than in MDA468. In the latter cell line, treatment with SAHA alone or in combination with PJ34 affected cell proliferation in a comparable manner.

To evaluate whether effects of the two drugs on cell viability were associated with modification of apoptosis, the caspase 3/7 activity was assessed (Fig. 5B). The MDA157 cell line exhibited an almost 2-fold increase in caspase 3/7 activity after $72 \mathrm{~h}$ SAHA treatment, and a 2.5 -fold after SAHA and PJ34 treatment, while a slight reduction following treatment with PJ34 alone was observed. In the MDA468 cell line, a mild increase in apoptosis with SAHA alone or in combination with 
A

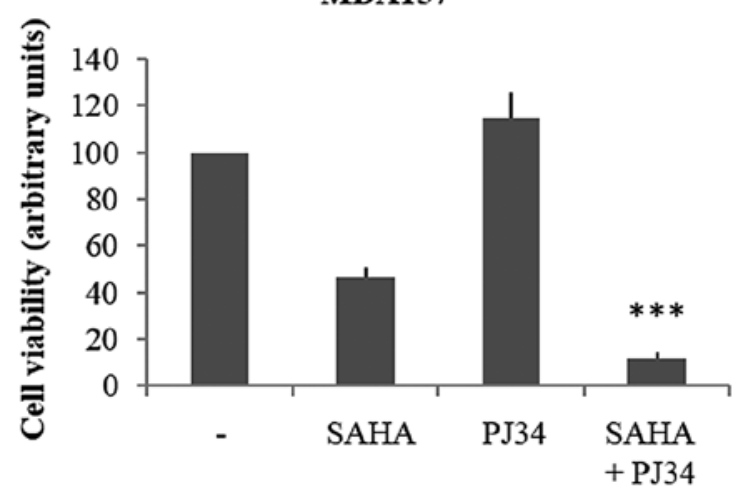

B

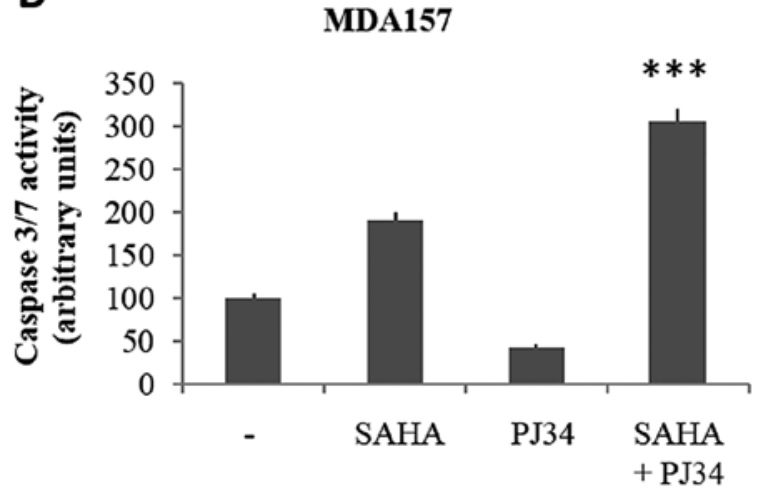

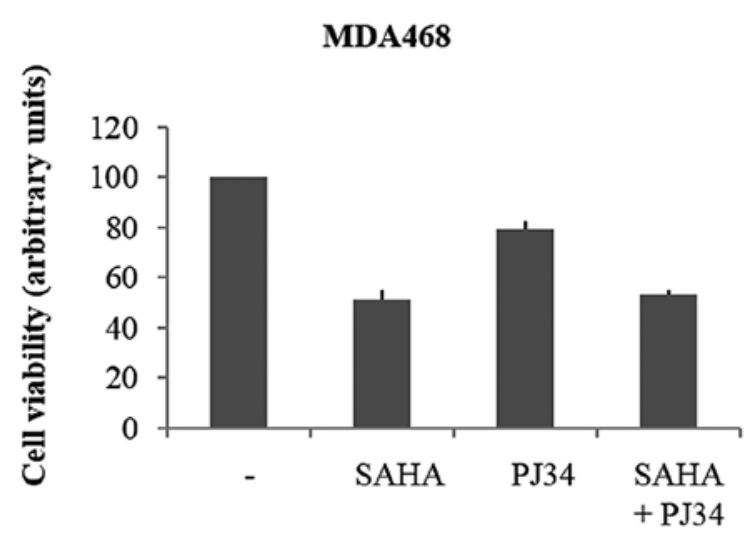

MDA468

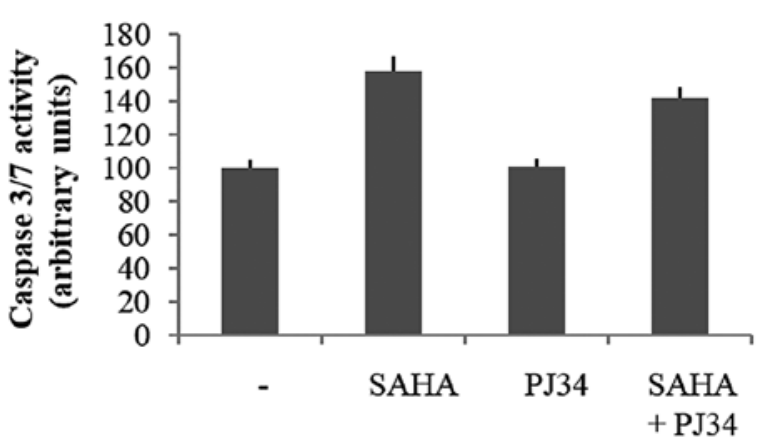

Figure 5. Effects of HDAC and PARP inhibitors on cell viability and apoptosis in MDA157 and MDA468 cells treated for 72 h with SAHA $3 \mu$ M, or PJ34 $5 \mu \mathrm{M}$ alone or in combination. (A) MTT assay was performed as described in Materials and methods. (B) Caspase 3/7 activity was measured as a marker of apoptosis. Each bar is the mean value $\pm \mathrm{SD}$ of three independent determinations. (A and $\mathrm{B})$ *Significant difference compared to all others conditions $(\mathrm{P}<0.05)$.

PJ34 was observed, while treatment with PJ34 alone produced no detectable effects. Therefore, treatment with SAHA and PJ34 in combination determines an increase in apoptotic processes in MDA157 but not in MDA468 cells.

Taken together, these results suggested that cells that are responsive to the SAHA and PJ34 combination, showing an increase of TXNIP protein levels, were also responsive in terms of cell growth parameters.

\section{Discussion}

For many years, cancer origin and progression have been recognized to be secondary to the accumulation of genetic mutations that may result in oncogene activation and tumor suppressor gene inactivation. The recent finding that reversible alterations in histone proteins and DNA can also lead to tumorigenesis has introduced a novel term in the field of cancer research known as 'epigenetics' (23). The importance of cancer epigenetics has led many investigators to incorporate this novel and noteworthy field in anticancer drug development. Several drugs that target epigenetic alterations, including inhibitors of HDAC and DNA methyltransferase (DNMT), are currently approved for the treatment of hematological malignancies and are available for clinical investigation in solid tumors (23). Epigenetic mechanisms may play a role in TXNIP gene expression. It appears that in cancer cells TXNIP expression is downregulated through epigenetic mechanisms, such as deacetylation and hypermethylation at the promoter level (6). Our data indicate that epigenetic marking on TXNIP gene in MDA157 and MDA468 cell lines is different. Histone post-translational modifications are an important regulatory platform for gene expression, replication, DNA repair, chromosome segregation and cell death (24). Specific histone modifications are associated with gene expression activation or silencing. Therefore, we investigated histone $\mathrm{H} 3$ lysine 4 trimethylation ( $\mathrm{H} 3 \mathrm{~K} 4 \mathrm{me} 3$ ), and histone $\mathrm{H} 3$ lysines 9 and 14 acetylation (H3K9K14ac), which are markers of active transcription, and histone H3 lysine 27 trimethylation (H3K27me3), which instead characterizes silenced genes. In MDA157 cells basal levels of H3K9K14ac, H3K4me3 and $\mathrm{H} 3 \mathrm{~K} 27 \mathrm{me} 3$ are measurable. This signature indicates that the TXNIP gene, in these cells, was paused, and likely to be activated by exogenous stimulation. Treatment with SAHA and $\mathrm{PJ} 34$ reduced the levels of $\mathrm{H} 3 \mathrm{~K} 27 \mathrm{me} 3$, while maintaining high levels of $\mathrm{H} 3$ acetylation, resulting in an increased TXNIP expression. Conversely, in MDA468 cells, the TXNIP gene was definitely silenced, with significant levels of $\mathrm{H} 3 \mathrm{~K} 27 \mathrm{me} 3$ being only observable in the basal state and increased following the combined treatment of SAHA and PJ34. Thus, we showed that the model of bivalent marking may accurately predict the cell responsiveness of TXNIP gene following the combined treatment of SAHA and PJ34. In MDA157 cells the pausing signature was permissive for the synergy between SAHA and PJ34. Epigenetic profiling of cancer cells may reveal 
oncosuppressor genes that are paused and that, therefore, may be activated by pharmacological treatment.

The synergy between SAHA and PJ34 observed in MDA157 cells was of great interest in terms of cancer treatment. A central issue in the development of anticancer compounds was to increase the therapeutic index, limiting at the same time, the development of resistance. One solution was to combine multiple drugs that act synergistically. A number of ongoing clinical trials are investigating the effects of combined therapy against different types of cancer (25-27). In our experimental system, the effect of combined treatment with SAHA and PJ34 on TXNIP expression was associated with variation in cell viability and apoptosis. In the MDA157 cell line, the increase of TXNIP expression was associated with a decrease in cell viability and an increase in apoptosis. By contrast, MDA468 cells were not responsive to the SAHA-PJ34 cocktail, both in terms of TXNIP expression and cell proliferation parameters. These findings are consistent with those of previous studies, in which a correlation between TXNIP expression increment, growth inhibition and induction of apoptosis has been shown $(19,22)$. Moreover, Jasek et al have recently shown that the combined treatment of SAHA and PJ34 on leukemic cell lines has a synergistic effect on proliferation inhibition and an increase in apoptosis (28). Thus, our data support the hypothesis that TXNIP is an effective target for the treatment of breast cancer.

\section{Acknowledgements}

This study is funded by grants to GD from Associazione Italiana per la Ricerca sul Cancro (AIRC) (project no. IG 10296).

\section{References}

1. Chen KS and DeLuca HF: Isolation and characterization of a novel cDNA from HL-60 cells treated with 1,25-dihydroxyvitamin D-3. Biochim Biophys Acta 1219: 1226-1232, 1994.

2. Kaimul AM, Nakamura $\mathrm{H}$, Masutani $\mathrm{H}$ and Yodoi J: Thioredoxin and thioredoxin-binding protein-2 in cancer and metabolic syndrome. Free Radic Biol Med 43: 861-868, 2007.

3. Elgort MG, O'Shea JM, Jiang Y and Ayer DE: Transcriptional and translational downregulation of thioredoxin interacting protein is required for metabolic reprogramming during $\mathrm{G}(1)$. Genes Cancer 1: 893-907, 2010.

4. Patwari P, Chutkow WA, Cummings K et al: Thioredoxinindependent regulation of metabolism by the alpha-arrestin proteins. J Biol Chem 284: 24996-25003, 2009.

5. Spindel ON, World C and Berk BC: Thioredoxin interacting protein: redox dependent and independent regulatory mechanisms. Antioxid Redox Signal 16: 587-596, 2012.

6. Zhou J, Yu Q and Chng WJ: TXNIP (VDUP-1, TBP-2): a major redox regulator commonly suppressed in cancer by epigenetic mechanisms. Int J Biochem Cell Biol 43: 1668-1673, 2011.

7. Cadenas C, Franckenstein D, Schmidt M, et al: Role of thioredoxin reductase 1 and thioredoxin interacting protein in prognosis of breast cancer. Breast Cancer Res 12: R44, 2010.
8. Frullanti E, Colombo F, Falvella FS, et al: Association of lung adenocarcinoma clinical stage with gene expression pattern in noninvolved lung tissue. Int J Cancer 131: E643-E648, 2012.

9. Nishizawa K, Nishiyama H, Matsui Y, et al: Thioredoxininteracting protein suppresses bladder carcinogenesis. Carcinogenesis 32: 1459-1466, 2011.

10. Butler LM, Zhou X, Xu WS, et al: The histone deacetylase inhibitor SAHA arrests cancer cell growth, up-regulates thioredoxin-binding protein-2, and down-regulates thioredoxin. Proc Natl Acad Sci USA 99: 11700-11705, 2002.

11. Ku WL, Girvan M, Yuan GC, Sorrentino F and Ott E: Modeling the dynamics of bivalent histone modifications. PLoS One 8: e77944, 2013

12. Santos-Rosa H, Schneider R, Bannister AJ, et al: Active genes are tri-methylated at K4 of histone H3. Nature 419: 407-411, 2002.

13. Barski A, Cuddapah S, Cui K, et al: High-resolution profiling of histone methylations in the human genome. Cell 129: 823-837, 2007.

14. Chavez KJ, Garimella SV and Lipkowitz S: Triple negative breast cancer cell lines: one tool in the search for better treatment of triple negative breast cancer. Breast Dis 32: 35-48, 2010.

15. Lavarone E, Puppin C, Passon N, Filetti S, Russo D and Damante G: The PARP inhibitor PJ34 modifies proliferation, NIS expression and epigenetic marks in thyroid cancer cell lines. Mol Cell Endocrinol 365: 1-10, 2013.

16. Baldan F, Lavarone E, Di Loreto C, Filetti S, Russo D, Damante G and Puppin C: Histone post-translational modifications induced by histone deacetylase inhibition in transcriptional control units of NIS gene. Mol Biol Rep 41: 5257-5265; 2014.

17. Marks PA and Xu WS: Histone deacetylase inhibitors: potential in cancer therapy. J Cell Biochem 107: 600-608, 2009.

18. Russo D, Damante G, Puxeddu E, Durante C and Filetti S: Epigenetics of thyroid cancer and novel therapeutic targets. J Mol Endocrinol 46: R73-R81, 2011.

19. Zhou J and Chng WJ: Roles of thioredoxin binding protein (TXNIP) in oxidative stress, apoptosis and cancer. Mitochondrion 13: 163-169, 2013.

20. Ungerstedt JS, Sowa Y, Xu WS, et al: Role of thioredoxin in the response of normal and transformed cells to histone deacetylase inhibitors. Proc Natl Acad Sci USA 102: 673-678, 2005.

21. Chen A: PARP inhibitors: its role in treatment of cancer. Chin J Cancer 30: 463-471, 2011.

22. Yamaguchi F, Hirata Y, Akram H, et al: FOXO/TXNIP pathway is involved in the suppression of hepatocellular carcinoma growth by glutamate antagonist MK-801. BMC Cancer 13: 468, 2013.

23. Connolly R and Stearns V: Epigenetics as a therapeutic target in breast cancer. J Mammary Gland Biol Neoplasia 17: 191-204, 2012.

24. Füllgrabe J, Kavanagh E and Joseph B: Histone onco-modifications. Oncogene 30: 3391-3403, 2011.

25. Lee JM, Hays JL, Annunziata CM, et al: Phase I/Ib study of olaparib and carboplatin in BRCA1 or BRCA2 mutation-associated breast or ovarian cancer with biomarker analyses. J Natl Cancer Inst: May 19, 2014 (Epub ahead of print). doi: 10.1093/ jnci/dju089.

26. Chu BF, Karpenko MJ, Liu Z, et al: Phase I study of 5-aza-2'-deoxycytidine in combination with valproic acid in non-small-cell lung cancer. Cancer Chemother Pharmacol 71: 115-121, 2013.

27. Falchook GS, Fu S, Naing A, et al: Methylation and histone deacetylase inhibition in combination with platinum treatment in patients with advanced malignancies. Invest New Drugs 31: 1192-1200, 2013

28. JasekE, Gajda M,Lis GJ, Jasińska Mand Litwin JA: Combinatorial effects of PARP inhibitor PJ34 and histone deacetylase inhibitor vorinostat on leukemia cell lines. Anticancer Res 34: 1849-1856, 2014. 